Subscriber access provided by KU Leuven Libraries

Communication

\title{
Controlling the Quality Factor of a Single Acoustic Nanoresonator by Tuning its Morphology
}

Fabio Medeghini, Aurélien Crut, Marco Gandolfi, Francesco Rossella, Paolo Maioli, Fabrice Vallée, Francesco Banfi, and Natalia Del Fatti

Nano Lett., Just Accepted Manuscript • DOI: 10.1021/acs.nanolett.8b02096 • Publication Date (Web): 10 Jul 2018

Downloaded from http://pubs.acs.org on July 11, 2018

\section{Just Accepted}

"Just Accepted" manuscripts have been peer-reviewed and accepted for publication. They are posted online prior to technical editing, formatting for publication and author proofing. The American Chemical Society provides "Just Accepted" as a service to the research community to expedite the dissemination of scientific material as soon as possible after acceptance. "Just Accepted" manuscripts appear in full in PDF format accompanied by an HTML abstract. "Just Accepted" manuscripts have been fully peer reviewed, but should not be considered the official version of record. They are citable by the Digital Object Identifier (DOI®). "Just Accepted" is an optional service offered to authors. Therefore, the "Just Accepted" Web site may not include all articles that will be published in the journal. After a manuscript is technically edited and formatted, it will be removed from the "Just Accepted" Web site and published as an ASAP article. Note that technical editing may introduce minor changes to the manuscript text and/or graphics which could affect content, and all legal disclaimers and ethical guidelines that apply to the journal pertain. ACS cannot be held responsible for errors or consequences arising from the use of information contained in these "Just Accepted" manuscripts. 


\title{
Controlling the Quality Factor of a Single Acoustic Nanoresonator by Tuning its Morphology
}

\author{
Fabio Medeghini ${ }^{1}$, Aurélien Crut ${ }^{1^{*}}$, Marco Gandolfi ${ }^{2,3,4}$, Francesco Rossella ${ }^{5}$, Paolo Maioli ${ }^{1}$, Fabrice \\ Vallée $^{1}$, Francesco Banfi ${ }^{2}$ and Natalia Del Fatti ${ }^{1}$ \\ ${ }^{1}$ FemtoNanoOptics group, Université de Lyon, CNRS, Université Claude Bernard Lyon 1, Institut \\ Lumière Matière, F-69622 Villeurbanne, France \\ 2 Interdisciplinary Laboratories for Advanced Materials Physics (I-LAMP), Università Cattolica del \\ Sacro Cuore, Brescia I-25121, Italy \\ ${ }^{3}$ Dipartimento di Matematica e Fisica, Università Cattolica del Sacro Cuore, Brescia I-25121, Italy \\ ${ }^{4}$ Laboratory of Soft Matter and Biophysics, Department of Physics and Astronomy, KU Leuven, \\ Celestijnenlaan 200D, B-3001 Heverlee, Leuven, Belgium \\ ${ }^{5}$ NEST, Scuola Normale Superiore and Istituto Nanoscienze-CNR, Piazza S. Silvestro 12, I-56124 Pisa, \\ Italy
}

\section{ABSTRACT:}

The mechanical vibrations of individual gold nanodisks nanopatterned on a sapphire substrate are investigated using ultrafast time-resolved optical spectroscopy. The number and characteristics of the detected acoustic modes are found to vary with nanodisk geometry. In particular, their quality factors strongly depend on nanodisk aspect ratio (i.e., diameter over height ratio), reaching a maximal value of $\approx 70$, higher than those previously measured for substrate-supported nanoobjects. The peculiarities of the detected acoustic vibrations are confirmed by finite-element simulations, and interpreted as the result of substrate-induced hybridization between the vibrational modes of a nanodisk. The present findings demonstrate novel possibilities for engineering the vibrational modes of nano-objects.

KEYWORDS: Nano-objects, vibrations, ultrafast, damping, substrate, hybridization 
The vibrational properties of nano-objects currently attract great interest, motivated by both fundamental questions and application possibilities. Their study provides information on the laws governing elasticity and energy transfer at the nanoscale. ${ }^{1-4}$ Moreover, their high sensitivity to mass deposition, resulting from the small mass of nano-objects and their high vibrational frequencies, makes them promising for mass sensing applications. ${ }^{5-12}$ Nano-object vibrations can be experimentally addressed using optics-based methods such as Raman and time-resolved spectroscopies, which enable the detection of a few vibrational modes and the measurement of their frequencies and, under certain conditions, of their decay rates. ${ }^{13-18}$ Experiments performed in the last twenty years in this field have clarified the dependence of vibrational frequencies on nano-object size, shape, crystallinity and environment. ${ }^{1,2,19-22}$ In particular, their surprisingly accurate reproduction by continuum mechanics models, even in the case of ultrasmall $(\sim 1 \mathrm{~nm})$ nanoparticles, ${ }^{23,24}$ has been repeatedly demonstrated. However, many questions still remain open regarding the nature and efficiency of the mechanisms ruling the vibrational damping of nano-objects. In contrast with vibrational frequencies which are predominantly determined by intrinsic nano-object properties (e.g., composition, morphology and crystallinity), ${ }^{1}$ decay rates are very sensitive to the properties of the nanoobject/environment interface, which determine the efficiency with which acoustic waves are emitted in the environment. Additionally, a quantitative investigation of damping based on measurements on ensembles of nanoparticles is challenging because of the spurious inhomogeneous effects (i.e., the fact that nanoparticles in an assembly vibrate at different frequencies due to the dispersion of their morphology) affecting these experiments. Up to now, investigations of vibrational damping on ensembles of nano-objects have thus been limited to two types of nano-objects that could be synthesized with particularly low morphological dispersion (silver nanospheres embedded in glass ${ }^{15}$ and gold bipyramids in solution $\left.{ }^{4,25}\right)$.

The development of time-resolved experiments on single nano-objects a decade ago has paved the way to a more detailed investigation of vibrational damping, allowing direct measurement of the frequency $f$, decay rate $\Gamma$ and quality factor $Q=\pi f / \Gamma$ associated to the detected vibrational modes. ${ }^{26-29}$ In such experiments, the value of $Q$ is limited by two distinct categories of damping processes, namely radiative and intrinsic damping. The former mechanism refers to the conversion of localized nano-object vibrations into propagative acoustic waves in its environment by elastic energy transfer through the nano-object surface. Its associated quality factor, $\mathrm{Q}_{\mathrm{env}}$, strongly depends on acoustic mismatch ${ }^{19}$ and mechanical contact quality ${ }^{7,10,30-35}$ at the interface between the nano-object and its local environment. The 
details of the latter mechanism, associated to a quality factor $Q_{i n t}$, remain to be understood. Assuming the two mechanisms to be independent leads to the following expression for $Q$ :

$$
\frac{1}{\mathrm{Q}}=\frac{1}{\mathrm{Q}_{\mathrm{env}}}+\frac{1}{\mathrm{Q}_{\mathrm{int}}}
$$

Acoustic coupling with the environment is the dominant damping mechanism in experiments performed on substrate-deposited nano-objects synthesized by chemical methods. ${ }^{1,28,29,36} \mathrm{Q}$ values in the 5-50 range were measured for their detected vibrational modes, with a large interparticle dispersion, attributed to variations of the quality of the nanoparticle/substrate mechanical contact. Lower quality factors have been recently reported for lithographed nano-objects, ${ }^{37-39}$ resulting from lower $Q_{\text {int }}$ values as compared to chemically synthesized ones. Experiments on nanowires suspended over a trench have the advantage to enable an experimental estimation of both $Q_{\text {env }}$ and $Q_{\text {int }}$ by comparing the acoustic behaviour of the same nanowire in air (where $Q \approx Q_{\text {int }}$ ) and liquid environments (where $Q$ is given by Eq. 1). ${ }^{40-42}$ As compared to nanoparticles, interpretation of the extracted $Q_{i n t} \approx 100$ values is however complicated by the occurrence of an additional phenomenon, i.e. the propagation of acoustic waves along the nanowire away from its excited part. ${ }^{42}$ This overview highlights the still limited understanding of nano-object vibrational damping. In this paper, we demonstrate that the vibrational quality factors of substrate-supported nano-objects strongly depend on their morphology, and may be significantly enhanced for specific shape choices. To this end, systematic time-resolved studies were performed on individual gold nanodisks (NDs) nanopatterned on a sapphire substrate, chosen because of their potential technological relevance and of their shape, described by only two lengths, i.e., their diameter $D$ and height $h$ (with their aspect ratio defined as $\eta=D / h$ ) and allowing a large contact area with the substrate.

Gold NDs with a diameter spanning the $\mathrm{D}=60-200 \mathrm{~nm}$ range and $\mathrm{h} \approx 20$ or $40 \mathrm{~nm}$ thickness were nanopatterned by electron beam lithography (EBL) and lift-off techniques on the optically polished surface of a $480 \mu \mathrm{m}$ thick (0001) $\alpha-\mathrm{Al}_{2} \mathrm{O}_{3}$ single crystal (sapphire) substrate. The adopted procedures grant both a good morphology control and a clean disk/substrate interface. A large $(10 \mu \mathrm{m})$ separation between NDs was chosen so as to allow optical investigation of a single ND and to avoid the acoustic cross-talk between the NDs occurring in phononic crystals ${ }^{12,43}$ and oligomers of close NDs. ${ }^{44}$ Several replicas of the above mentioned samples were built (in multiple deposition sessions) and measured so as to rule-out possible spot contamination and evaporation session-dependent effects. Atomic force microscopy (AFM) and scanning electron microscopy (SEM) characterizations of the NDs were 
performed for a first selection of the best quality NDs. Complementary information on the circular character of the NDs was obtained by measuring their linear optical response using spatial modulation spectroscopy (SMS). ${ }^{45-48}$ This single-particle technique is based on the periodic displacement of a single nano-object in the focal spot of a tightly focused light beam, which induces a modulation of the transmitted light power (Fig. 1a). It enables the quantitative determination of the nano-object extinction cross-section $\sigma_{\mathrm{ext}}$ as a function of the illumination wavelength $\lambda$ and light polarization angle $\theta$. SMS experiments were performed using a tunable Ti:sapphire oscillator combined with a visible optical parametric oscillator as light source, allowing ND optical characterization in the 540-1040 nm wavelength range. The light beam delivered by this source was focused down to the diffraction limit (about $0.7 \lambda$ full-width at half-maximum) on a sample by a 100X microscope objective, the direction of its linear polarization being controlled by a wire grid polarizer. Spatial modulation of the sample was performed at $\mathrm{f}=1.5 \mathrm{kHz}$ frequency and lock-in detection at $2 \mathrm{f}$. The dependence of $\sigma_{\text {ext }}$ on the incident light polarization direction $\theta$ shows two distinct behaviors among the individual NDs of the produced samples. For a fraction of them, $\sigma_{\text {ext }}$ is almost independent of $\theta$, and its spectrum exhibits a quasi-Lorentzian peak associated to the ND dipolar localized surface plasmon resonance (SPR) (Fig. 1b-c). ${ }^{47,49,50}$ This behavior corresponds to the response expected for an ideal ND shape. Conversely, for many other NDs, marked variations of $\sigma_{\text {ext }}$ with $\theta$ occur, indicating a non-circular ND section, and leading to a ND acoustic response more complex and difficult to interpret. Such nano-objects were discarded in the present study. Fig. $1 \mathrm{~d}$ shows the spectral position of SPR for the selected circular $h=40 \mathrm{~nm}$ NDs, which linearly red-shifts for increasing ND aspect ratio. Such linear evolution is in agreement with the results of finiteelement simulations including the inhomogeneous ND environment (air and sapphire substrate) (Fig. 1d). The measured and modelled dependences however present a small shift, corresponding to an aspect ratio difference of 0.2-0.3. Such behaviour presumably results from the fabrication of NDs either cylindrical with a diameter slightly smaller (by about $10 \mathrm{~nm}$ ) than that the nominal ones, or slightly conical, i.e. narrowing at their top (as reported in ref. ${ }^{37}$ ).

Time-resolved experiments on individual NDs (Fig. 1e) were then performed by combining the SMS microscope with a two-color pump-probe setup based on the previously described femtosecond laser source, delivering $150 \mathrm{fs}$ pulses. The oscillator pulse train was split in two parts to generate the pump and probe beams, a different wavelength being generated for one of them using either second harmonic generation or the optical parameter oscillator. Incident pump fluences of a few hundreds of $\mu \mathrm{J} . \mathrm{cm}^{-2}$ were typically used. The relative changes of probe beam transmission, $\Delta T / T$, were measured as a function of the time 
interval separating pump and probe pulses, controlled by a mechanical delay line. The timeresolved signal measured for a ND with $D=150 \mathrm{~nm}$ and $h=40 \mathrm{~nm}$ (i.e., $\eta=3.75$ ) using a $\lambda=950$ $\mathrm{nm}$ probe wavelength is shown as an example in Fig. 1f. It contains signatures of the creation of an athermal electronic distribution by pump pulse absorption and the induced relaxation mechanisms. The peak observed at short timescales reflects the ND ultrafast excitation and the internal gold thermalization on $a \approx 1$ ps timescale (by electron-electron and electronphonon scattering mechanisms, process 1 in Fig. 1f). ${ }^{11}$ This impulsively launches ND acoustic vibrations (by a displacive excitation mechanism induced by thermal dilation), causing damped oscillations of time-resolved signals on nanosecond timescales (process 2 in Fig. 1f). ${ }^{1}$ This feature partly overlaps with a monotonic decay of the signal associated to ND cooling ${ }^{52}$ (i.e., dissipation of the thermal energy injected by the pump pulse also occurring on a nanosecond timescale, process 3 in Fig. 1f).

The oscillating components of the measured time-resolved signals were isolated by subtraction of the internal thermalization and cooling contributions (Fig. 2). Each of the resulting signals was then fitted by one or two (depending on ND aspect ratio $\eta$ ) damped sinusoids, each associated to the contribution of a specific vibrational mode, allowing determination of its frequency, decay rate (and thus quality factor) and amplitude. The oscillating signals obtained for $\eta>3.2$ could be well fitted by a single damped sinusoid, corresponding to the detection of a single vibrational mode (the $\eta=3.5$ case is shown in Fig. 2a). Conversely, for $2.2<\eta<3.2$, the oscillating component displayed a beating pattern (particularly clear in the $\eta=3$ case shown in Fig. $2 b$ ) which could be successfully reproduced by considering two modes of close frequencies in the analysis. Finally, for $1.5<\eta<2.2$, two modes with frequencies differing by a factor of about 2 were detected (Fig. $2 d$, corresponding to $\eta=2$ ). The conclusions of these time-domain analyses on single NDs of different morphologies were confirmed by calculating the energy spectral density (ESD) of the oscillating components (insets of Fig. 2), presenting a single peak for $\eta=3.5$ (Fig. 2a), two overlapping ones for $\eta=3$ (Fig. 2b) and two well-separated ones for $\eta=2$ (Fig. 2d).

A striking result of these investigations is the large variation of vibrational lifetimes and quality factors with $\eta$. This effect is clearly visible in Fig. 2 , where the signal obtained for $\eta=2.5$ (Fig. 2c) presents a much larger number of oscillations than the other ones shown in this figure. It is further illustrated in Fig. S1 of the Supporting Information, which compares oscillating signals measured for a larger variety of $\eta$ values. Figs. 2 and S1 show large variations of the number of oscillations occurring before mechanical equilibrium, i.e. of the highest quality factor detected for each $\eta$ value, shown in Fig. 3a. In particular, measurements show a 
dramatic enhancement of this quality factor around $\eta \approx 2.5$, which reaches $Q \approx 70$, a value larger than the 5-50 ones typically obtained for substrate-supported nano-objects. ${ }^{1} \mathrm{~A}$ second, less pronounced $Q$ enhancement $(Q \approx 30)$ occurs near $\eta=6$ over a broader $\eta$ range.

A complete summary of the measured characteristics of the detected modes is presented in Fig. $4 a-b$ as a function of ND aspect ratio. The measured frequencies are shown in these two panels, while the corresponding Q factors (Fig. 4a) and integrated ESDs (Fig. 4b, associated to the efficiencies with which vibrational modes are excited and detected in the context of timeresolved experiments) are presented through colour scales. In contrast with the sharp variations of quality factors with morphology changes, the measured frequencies smoothly vary with $\eta$, either slightly increasing with $\eta$ for the low-frequency mode detected below $\eta=2.2$ or decreasing throughout the full $\eta$ range explored for the other modes. The low-frequency mode detected below $\eta=2.2$ has a quality factor $(Q \approx 5)$ lower than the high frequency one $(Q \approx 15)$ also detected in this range (Fig. 4a). The two modes observed in the intermediate $\eta$ range present close frequencies but very different quality factors (Fig. 4a, with $Q<10$ for the lower-frequency mode, and $Q=10-70$, depending on $\eta$, for the higher-frequency one).

Finite-element modeling (FEM) was performed to identify the detected modes and numerically estimate their frequencies and quality factors, as well as the efficiency with which they are excited in the context of ultrafast time-resolved experiments (i.e., by quasi-uniform initial heating). The FEM model was built by adapting the strategy to include nano-object environment described in a recent work $^{53}$ to the case of our experimental configuration (see the text and Fig. S2 of the Supporting Information). Simulations were performed in the frequency domain, vibrational spectra being obtained by computing the average elastic energy stored in a ND as a function of the frequency of a periodical excitation (a uniform stress corresponding to the experimental case of a homogeneously excited ND being chosen). A perfect mechanical contact (i.e., continuity of displacement and normal stress) was assumed at the ND/substrate interface, and stress-free boundary conditions were used on the other ND faces. No intrinsic acoustic damping mechanism was considered in the ND and its environment (i.e., infinite $Q_{\text {int }}$, so that the simulated quality factors correspond to $Q_{\text {env }}$ ).

Fig. 4c-d presents the frequencies, quality factors and normalized spectral areas (by division by the maximal value obtained among the modes shown in the plot) extracted from the excitation vibrational spectra shown in Figs. S3 and S4 of the Supporting Information (only the two or three lowest frequency modes relevant for the analysis of experimental results are shown in these figures; the highest quality factor among these modes for each $\eta$ value is also 
shown in Fig. 3b). FEM simulations lead to a quantitative reproduction of the measured frequencies, thus allowing access to the displacement field of the detected modes (Fig. 5). Furthermore, they show that the partition of elastic energy among the ND modes dramatically varies with $\eta$ (Fig. 4d), explaining why some of these modes become undetectable for some aspect ratios (e.g., the $10 \mathrm{GHz}$ low-frequency mode for $\eta>2.5$; note that only a qualitative comparison between the experimental and simulated mode areas respectively shown in Fig. $4 \mathrm{~b}$ and $4 \mathrm{~d}$ is possible, as simulations do not take into account the efficiency with which each mode is optically detected). The only oscillation detected in the high $\eta$ range $(\eta>3.2)$ is ascribed to a mode involving a non-uniform modification of both ND height and diameter (mode $1 a$ in Fig. 5a). A mode involving ND bending also appears in simulations at a slightly larger frequency (mode $2 a$ in Fig. 5a). Its computed spectral area decreases with increasing $\eta$ (Fig. 4d), explaining why it could not be experimentally observed for $\eta>3.2$. However, for $\eta \approx 3$, the frequencies and excitation efficiencies of these two modes get closer, explaining the beating pattern observed in the time-resolved signal of Fig. $2 \mathrm{~b}$. In the low $\eta$ range $(\eta \leq 2.5)$, FEM simulations yield three relevant modes, whose displacement profiles are presented in Fig. $5 b$ for $\eta=2.5$. The two highest frequency ones (modes $2 b$ and $3 b$ in Fig. $5 b$ ) are however predicted to be excited with very different efficiencies for most $\eta$ values (Fig. 4d), explaining the detection of only two modes with largely different frequencies in this low $\eta$ regime (Fig. 2d).

The most important result of the presented FEM simulations is the large $\eta$ dependence of vibrational quality factors that they predict (Figs. 3b and 4c). This dependence is in excellent agreement with experimental trends, also presenting large enhancement of the maximal quality factor near $\eta=2.5$, as well as a smaller and broader one near $\eta=6$ (Figs. 3a and 4a). The dependence of vibrational quality factors of substrate-supported nano-objects on their morphology can be qualitatively interpreted as the result of the hybridization of the ND normal vibrational modes induced by the presence of the substrate. Such hybridization leads to the loss of a well-defined (e.g., translational, quadrupolar-like or bending) character for vibrational modes, generating more complex displacement profiles (Fig. 5). The displacement and strain profiles at the ND/substrate interface, which rule the efficiency with which acoustic energy is radiated in the substrate by the ND, sensitively depend on the displacement fields of the normal modes being hybridized (and thus of the ND aspect ratio, the parameter varied in this study), on the strength of their interaction (depending on the acoustic mismatch at the $\mathrm{ND} /$ substrate, here fixed), and on the considered hybridized mode. As a result, hybridization produces modes with very different acoustic radiation efficiencies, generating poor quality 
factors in some cases (such as that of mode $1 b$ of Fig. $5 b$, for which $Q_{e n v}=2$ ) but also producing quasi-localized vibrational modes, effectively isolated from the substrate for specific modes and morphologies.

The present study thus reveals new facets of vibrational coupling (i.e., substrate-mediated hybridization between different acoustic modes of the same nano-object) as compared to recent studies which addressed the interactions between vibrational modes of different nanoobjects assembled as dimers ${ }^{53,56}$ or oligomers, ${ }^{44}$ also showing that they induce frequency shifts and quality factor modifications. The substrate-mediated hybridization between the acoustic modes of a ND evoked here also presents a strong analogy with that affecting the plasmonic modes of nano-objects in strong electromagnetic interaction with a substrate, such as for instance silver nanocubes deposited on glass. In this case, substrate deposition of the nanocubes electromagnetically couples their dipolar and quadrupolar plasmon modes (respectively bright and dark, i.e. strongly and weakly coupled to light in the absence of substrate), producing two bonding and antibonding modes which are both detectable in the scattering spectra of single nanocubes. ${ }^{57,58}$

The main difference between experimental results and those of the complete FEM simulations is the absolute value of the quality factors. In particular, the experimental maximal $Q$ value is smaller than the computed one by a $>50$ factor (Fig. 3). This discrepancy could result from either an imperfect mechanical contact between the NDs and the substrate or from ND intrinsic damping (i.e., the $\mathrm{Q}_{\text {int }}$ term in eq. 1 ), two effects not included in the simulations.

The former hypothesis, related to the ND/substrate interface, is however unlikely, as the assumption in the modelling of a good mechanical contact at this interface is essential to reproduce the large quality factor variations with $\eta$ (conversely, assuming a complete mechanical disconnection at the $N D /$ substrate interface would yield a $\eta$-independent $Q=Q_{\text {int }}$ value). To confirm the weak influence of such interface effects, we experimentally and numerically investigated the effect of adding a $4 \mathrm{~nm}$ thick chromium adhesion layer between the NDs and their substrate, finding little modification of the measured quality factors (note however that time-resolved experiments are more challenging in this case, due to the SPR broadening induced by the adhesion layer ${ }^{59}$ which reduces the sensitivity of the optical signal to vibrations). This suggests that a gold-sapphire interface still enables an efficient transmission of acoustic waves from the NDs to the substrate, even in the absence of an adhesion layer. 
Conversely, the hypothesis that the vibrational quality factors of NDs are significantly affected by intrinsic damping is supported by the fact that electron lithography typically produces nano-objects presenting many crystalline defects, ${ }^{60,61}$ which are a significant source of intrinsic damping. ${ }^{22}$ In two recent studies on gold and aluminium NDs, intrinsic damping was even demonstrated to be the dominant source of vibrational damping (i.e., $Q_{i n t}<Q_{e n v}$ in eq. 1 ), leading to low $(\approx 10) Q$ values independent of the ND/substrate interface properties. ${ }^{38,39}$ This conclusion however differs from that drawn from studies on gold nanorings produced by colloidal lithography, where the acoustic radiation in the substrate was shown to be the dominant damping mechanism ${ }^{62}$, suggesting that the $Q_{\text {int }}$ value may strongly depend on the details of the fabrication procedure. Here, assuming that the difference between the maximal experimental and computed $Q$ values obtained for $\eta \approx 2.5$ solely results from intrinsic damping and that $Q_{\text {env }}$ is accurately described by numerical simulations, $Q_{\text {int }} \approx Q \approx 70$ is obtained for the NDs showing the largest $Q$ values ( $1 / Q_{\text {env }}$ being then negligible in eq. 1 for this aspect ratio). More generally, a good agreement between our experimental and modeled $Q$ values could be obtained for all investigated NDs using $Q_{\text {int }}$ values in the 40-70 range.

In conclusion, we have demonstrated both experimentally and through numerical simulations that the vibrational quality factors metal nano-objects on a substrate depend on their morphology, and that high quality factors can be achieved for specific shapes. Our study shows that an accurate choice of nano-object morphology can almost suppress its acoustic emission to the substrate, i.e. produce localized mechanical modes whose lifetimes are only limited by intrinsic damping mechanisms, enabling a detailed investigation of these phenomena. Minimization of the environment and intrinsic damping mechanisms, by tuning nano-object morphology and composition respectively, opens new perspectives for investigations of energy storage and transfer at ultrafast timescales and for nanosensing applications (e.g., nanobalances) based on the vibrations of substrate-supported single metal nano-objects.

\section{ASSOCIATED CONTENT}

\section{Supporting Information}

The Supporting Information is available free of charge on the ACS Publications website.

Description of FEM simulations; oscillating components of time-resolved pump-probe transmission changes measured for a variety of ND aspect ratios (examples); FEM-computed excitation vibrational 
spectra for different ND aspect ratios; excitation vibrational spectrum computed for $\eta=2$ ND aspect ratio (zoom).

\section{AUTHOR INFORMATION}

\section{Corresponding author}

*E-mail: aurelien.crut@univ-lyon1.fr

ORCID

Aurélien Crut : 0000-0003-2185-709X

Marco Gandolfi : 0000-0001-7700-9255

Francesco Banfi : 0000-0002-7465-8417

Natalia Del Fatti : 0000-0002-8074-256X

Notes

The authors declare no competing financial interest.

\section{ACKNOWLEDGMENTS}

F.B., M.G. and F.R. acknowledge financial support from the MIUR Futuro in ricerca 2013 Grant in the frame of the ULTRANANO Project (Project No. RBFR13NEA4) and support from Universita Cattolica del Sacro Cuore through D.2.2 and D.3.1 grants. M.G. acknowledges financial support from Academische Stichting Leuven and FWO. The authors thank Mr. Stefano Calati for support on the experimental set-up. 


\section{REFERENCES}

(1) Crut, A.; Maioli, P.; Del Fatti, N.; Vallée, F. Acoustic Vibrations of Metal Nano-Objects: TimeDomain Investigations. Phys. Rep. 2015, 549, 1-43.

(2) Crut, A.; Maioli, P.; Del Fatti, N.; Vallée, F. Time-Domain Investigation of the Acoustic Vibrations of Metal Nanoparticles: Size and Encapsulation Effects. Ultrasonics 2015, 56, 98108.

(3) Major, T. A.; Lo, S. S.; Yu, K.; Hartland, G. V. Time-Resolved Studies of the Acoustic Vibrational Modes of Metal and Semiconductor Nano-Objects. J. Phys. Chem. Lett. 2014, 5, 866-874.

(4) Pelton, M.; Chakraborty, D.; Malachosky, E.; Guyot-Sionnest, P.; Sader, J. Viscoelastic Flows in Simple Liquids Generated by Vibrating Nanostructures. Phys. Rev. Lett. 2013, 111, 244502.

(5) Chaste, J.; Eichler, A.; Moser, J.; Ceballos, G.; Rurali, R.; Bachtold, A. A Nanomechanical Mass Sensor with Yoctogram Resolution. Nat. Nanotechnol. 2012, 7, 301-304.

(6) Cardinal, M. F.; Mongin, D.; Crut, A.; Maioli, P.; Rodríguez-González, B.; Pérez-Juste, J.; LizMarzán, L. M.; Del Fatti, N.; Vallée, F. Acoustic Vibrations in Bimetallic Au@Pd Core-shell Nanorods. J. Phys. Chem. Lett. 2012, 3, 613-619.

(7) Mongin, D.; Juvé, V.; Maioli, P.; Crut, A.; Del Fatti, N.; Vallée, F.; Sánchez-Iglesias, A.; PastorizaSantos, I.; Liz-Marzán, L. M. Acoustic Vibrations of Metal-Dielectric Core-Shell Nanoparticles. Nano Lett. 2011, 11, 3016-3021.

(8) Dacosta Fernandes, B.; Spuch-Calvar, M.; Baida, H.; Tréguer-Delapierre, M.; Oberlé, J.; Langot, P.; Burgin, J. Acoustic Vibrations of Au Nano-Bipyramids and Their Modification under Ag Deposition: A Perspective for the Development of Nanobalances. ACS Nano 2013, 7, 76307639.

(9) Lassagne, B.; Garcia-Sanchez, D.; Aguasca, A.; Bachtold, A. Ultrasensitive Mass Sensing with a Nanotube Electromechanical Resonator. Nano Lett. 2008, 8, 3735-3738.

(10) Guillon, C.; Langot, P.; Del Fatti, N.; Vallée, F.; Kirakosyan, A. S.; Shahbazyan, T. V.; Cardinal, T.; Treguer, M. Coherent Acoustic Vibration of Metal Nanoshells. Nano Lett. 2007, 7, 138-142.

(11) Nardi, D.; Zagato, E.; Ferrini, G.; Giannetti, C.; Banfi, F. Design of a Surface Acoustic Wave Mass Sensor in the $100 \mathrm{GHz}$ Range. Appl. Phys. Lett. 2012, 100, 253106.

(12) Nardi, D.; Travagliati, M.; Murnane, M. M.; Kapteyn, H. C.; Ferrini, G.; Giannetti, C.; Banfi, F. Impulsively Excited Surface Phononic Crystals: A Route Toward Novel Sensing Schemes. IEEE 
Sens. J. 2015, 15, 5142-5150.

(13) Duval, E.; Boukenter, A.; Champagnon, B. Vibration Eigenmodes and Size of Microcrystallites in Glass: Observation by Very-Low-Frequency Raman Scattering. Phys. Rev. Lett. 1986, 56, 2052-2055.

(14) Hodak, J. H.; Martini, I.; Hartland, G. V. Observation of Acoustic Quantum Beats in Nanometer Sized Au Particles. J. Chem. Phys. 1998, 108, 9210-9213.

(15) Del Fatti, N.; Voisin, C.; Chevy, F.; Vallée, F.; Flytzanis, C.; Vallée, F. Coherent Acoustic Mode Oscillation and Damping in Silver Nanoparticles. J. Chem. Phys. 1999, 110, 11484-11487.

(16) Hartland, G. V. Optical Studies of Dynamics in Noble Metal Nanostructures. Chem. Rev. 2011, $111,3858-3887$.

(17) Nardi, D.; Banfi, F.; Giannetti, C.; Revaz, B.; Ferrini, G.; Parmigiani, F. Pseudosurface Acoustic Waves in Hypersonic Surface Phononic Crystals. Phys. Rev. B 2009, 80, 104119.

(18) Nardi, D.; Travagliati, M.; Siemens, M. E.; Li, Q.; Murnane, M. M.; Kapteyn, H. C.; Ferrini, G.; Parmigiani, F.; Banfi, F. Probing Thermomechanics at the Nanoscale: Impulsively Excited Pseudosurface Acoustic Waves in Hypersonic Phononic Crystals. Nano Lett. 2011, 11, 41264133.

(19) Voisin, C.; Christofilos, D.; Del Fatti, N.; Vallée, F. Environment Effect on the Acoustic Vibration of Metal Nanoparticles. Phys. B 2002, 316-317, 89-94.

(20) Hu, M.; Wang, X.; Hartland, G. V.; Mulvaney, P.; Perez-Juste, J.; Sader, J. E.; Juste, J. P. Vibrational Response of Nanorods to Ultrafast Laser Induced Heating: Theoretical and Experimental Analysis. J. Am. Chem. Soc. 2003, 125, 14925-14933.

(21) Hartland, G. V. Coherent Excitation of Vibrational Modes in Metallic Nanoparticles. Annu. Rev. Phys. Chem. 2006, 57, 403-430.

(22) Goubet, N.; Yan, C.; Polli, D.; Portalès, H.; Arfaoui, I.; Cerullo, G.; Pileni, M.-P. Modulating Physical Properties of Isolated and Self-Assembled Nanocrystals through Change in Nanocrystallinity. Nano Lett. 2013, 13, 504-508.

(23) Juvé, V.; Crut, A.; Maioli, P.; Pellarin, M.; Broyer, M.; Del Fatti, N.; Vallée, F. Probing Elasticity at the Nanoscale: Terahertz Acoustic Vibration of Small Metal Nanoparticles. Nano Lett. 2010, $10,1853-1858$.

(24) Sauceda, H. E.; Mongin, D.; Maioli, P.; Crut, A.; Pellarin, M.; Del Fatti, N.; Vallée, F.; Garzón, I. 
L. Vibrational Properties of Metal Nanoparticles: Atomistic Simulation and Comparison with Time-Resolved Investigation. J. Phys. Chem. C 2012, 116, 25147-25156.

(25) Pelton, M.; Sader, J. E.; Burgin, J.; Liu, M.; Guyot-Sionnest, P.; Gosztola, D. Damping of Acoustic Vibrations in Gold Nanoparticles. Nat. Nanotechnol. 2009, 4, 492-495.

(26) van Dijk, M. A.; Lippitz, M.; Orrit, M. Detection of Acoustic Oscillations of Single Gold Nanospheres by Time-Resolved Interferometry. Phys. Rev. Lett. 2005, 95, 267406.

(27) Burgin, J.; Langot, P.; Del Fatti, N.; Vallée, F.; Huang, W.; El-Sayed, M. A. Time-Resolved Investigation of the Acoustic Vibration of a Single Gold Nanoprism Pair. J. Phys. Chem. C 2008, $112,11231-11235$.

(28) Staleva, H.; Hartland, G. V. Vibrational Dynamics of Silver Nanocubes and Nanowires Studied by Single-Particle Transient Absorption Spectroscopy. Adv. Funct. Mater. 2008, 18, 38093817.

(29) Ruijgrok, P. V; Zijlstra, P.; Tchebotareva, A. L.; Orrit, M. Damping of Acoustic Vibrations of Single Gold Nanoparticles Optically Trapped in Water. Nano Lett. 2012, 12, 1063-1069.

(30) Christofilos, D.; Voisin, C.; Del Fatti, N.; Vallée, F. Femtosecond Nonlinear Optical Spectroscopy of the Acoustic Vibration of Metal Nanoparticles Under High Pressure. High Press. Res. 2002, 22, 277-281.

(31) Crut, A.; Juvé, V.; Mongin, D.; Maioli, P.; Del Fatti, N.; Vallée, F. Vibrations of Spherical CoreShell Nanoparticles. Phys. Rev. B 2011, 83, 205430.

(32) Crimmins, T. F.; Maznev, A. A.; Nelson, K. A. Transient Grating Measurements of Picosecond Acoustic Pulses in Metal Films. Appl. Phys. Lett. 1999, 74, 1344-1346.

(33) Tas, G.; Loomis, J. J.; Maris, H. J.; Bailes, A. A.; Seiberling, L. E. Picosecond Ultrasonics Study of the Modification of Interfacial Bonding by Ion Implantation. Appl. Phys. Lett. 1998, 72, 22352237.

(34) Hettich, M.; Bruchhausen, A.; Riedel, S.; Geldhauser, T.; Verleger, S.; Issenmann, D.; Ristow, O.; Chauhan, R.; Dual, J.; Erbe, A.; et al. Modification of Vibrational Damping Times in Thin Gold Films by Self-Assembled Molecular Layers. Appl. Phys. Lett. 2011, 98, 261908.

(35) Fernandes, B. D.; Vilar-Vidal, N.; Baida, H.; Massé, P.; Oberlé, J.; Ravaine, S.; TreguerDelapierre, M.; Saviot, L.; Langot, P.; Burgin, J. Acoustic Vibrations of Core-Shell Nanospheres: Probing the Mechanical Contact at the Metal-Dielectric Interface. J. Phys. Chem. C 2018, 122, 
9127-9133.

(36) Zijlstra, P.; Tchebotareva, A. L.; Chon, J. W. M.; Gu, M.; Orrit, M. Acoustic Oscillations and Elastic Moduli of Single Gold Nanorods. Nano Lett. 2008, 8, 3493-3497.

(37) Chang, W.-S.; Wen, F.; Chakraborty, D.; Su, M.-N.; Zhang, Y.; Shuang, B.; Nordlander, P.; Sader, J. E.; Halas, N. J.; Link, S. Tuning the Acoustic Frequency of a Gold Nanodisk through Its Adhesion Layer. Nat. Commun. 2015, 6, 7022.

(38) Su, M.-N.; Dongare, P. D.; Chakraborty, D.; Zhang, Y.; Yi, C.; Wen, F.; Chang, W.-S.; Nordlander, P.; Sader, J. E.; Halas, N. J.; et al. Optomechanics of Single Aluminum Nanodisks. Nano Lett. 2017, 17, 2575-2583.

(39) Yi, C.; Su, M.-N.; Dongare, P.; Chakraborty, D.; Cai, Y.-Y.; Marolf, D. M.; Kress, R. N.; Ostovar, B.; Tauzin, L. J.; Wen, F.; et al. Polycrystallinity of Lithographically Fabricated Plasmonic Nanostructures Dominates Their Acoustic Vibrational Damping. Nano Lett. 2018, acs.nanolett.8b00559.

(40) Major, T. A.; Crut, A.; Gao, B.; Lo, S. S.; Del Fatti, N.; Vallée, F.; Hartland, G. V. Damping of the Acoustic Vibrations of a Suspended Gold Nanowire in Air and Water Environments. Phys. Chem. Chem. Phys. 2013, 15, 4169-4176.

(41) Belliard, L.; Cornelius, T. W.; Perrin, B.; Kacemi, N.; Becerra, L.; Thomas, O.; Toimil-Molares, M. E.; Cassinelli, M. Vibrational Response of Free Standing Single Copper Nanowire through Transient Reflectivity Microscopy. J. Appl. Phys. 2013, 114, 193509.

(42) Jean, C.; Belliard, L.; Cornelius, T. W.; Thomas, O.; Toimil-Molares, M. E.; Cassinelli, M.; Becerra, L.; Perrin, B. Direct Observation of Gigahertz Coherent Guided Acoustic Phonons in Free-Standing Single Copper Nanowires. J. Phys. Chem. Lett. 2014, 5, 4100-4104.

(43) Travagliati, M.; Nardi, D.; Giannetti, C.; Gusev, V.; Pingue, P.; Piazza, V.; Ferrini, G.; Banfi, F. Interface Nano-Confined Acoustic Waves in Polymeric Surface Phononic Crystals. Appl. Phys. Lett. 2015, 106, 21906.

(44) Yi, C.; Dongare, P. D.; Su, M.-N.; Wang, W.; Chakraborty, D.; Wen, F.; Chang, W.-S.; Sader, J. E.; Nordlander, P.; Halas, N. J.; et al. Vibrational Coupling in Plasmonic Molecules. Proc. Natl. Acad. Sci. 2017, 114, 11621-11626.

(45) Arbouet, A.; Christofilos, D.; Del Fatti, N.; Vallée, F.; Huntzinger, J.; Arnaud, L.; Billaud, P.; Broyer, M. Direct Measurement of the Single-Metal-Cluster Optical Absorption. Phys. Rev. Lett. 2004, 93, 127401. 
(46) Christofilos, D.; Blancon, J.-C.; Arvanitidis, J.; San Miguel, A.; Ayari, A.; Del Fatti, N.; Vallée, F. Optical Imaging and Absolute Absorption Cross Section Measurement of Individual NanoObjects on Opaque Substrates: Single-Wall Carbon Nanotubes on Silicon. J. Phys. Chem. Lett. 2012, 3, 1176-1181.

(47) Crut, A.; Maioli, P.; Del Fatti, N.; Vallée, F. Optical Absorption and Scattering Spectroscopies of Single Nano-Objects. Chem. Soc. Rev. 2014, 43, 3921-3956.

(48) Crut, A.; Maioli, P.; Vallée, F.; Del Fatti, N. Linear and Ultrafast Nonlinear Plasmonics of Single Nano-Objects. J. Phys. Condens. Matter 2017, 29, 123002.

(49) Kelly, K. L.; Coronado, E.; Zhao, L. L.; Schatz, G. C. The Optical Properties of Metal Nanoparticles: The Influence of Size, Shape, and Dielectric Environment. J. Phys. Chem. B 2003, 107, 668-677.

(50) Myroshnychenko, V.; Rodríguez-Fernández, J.; Pastoriza-Santos, I.; Funston, A. M.; Novo, C.; Mulvaney, P.; Liz-Marzán, L. M.; García de Abajo, F. J. Modelling the Optical Response of Gold Nanoparticles. Chem. Soc. Rev. 2008, 37, 1792-1805.

(51) Voisin, C.; Del Fatti, N.; Christofilos, D.; Vallée, F. Ultrafast Electron Dynamics and Optical Nonlinearities in Metal Nanoparticles. J. Phys. Chem. B 2001, 105, 2264-2280.

(52) Gandolfi, M.; Crut, A.; Medeghini, F.; Stoll, T.; Maioli, P.; Vallée, F.; Banfi, F.; Del Fatti, N. Ultrafast Thermo-Optical Dynamics of Plasmonic Nanoparticles. J. Phys. Chem. C 2018, 122, 8655-8666.

(53) Girard, A.; Gehan, H.; Crut, A.; Mermet, A.; Saviot, L.; Margueritat, J. Mechanical Coupling in Gold Nanoparticles Supermolecules Revealed by Plasmon-Enhanced Ultralow Frequency Raman Spectroscopy. Nano Lett. 2016, 16, 3843-3849.

(54) Dubrovskiy, V.; Morochnik, V. Natural Vibrations of a Spherical Inhomogeneity in an Elastic Medium. Izv. Earth Phys. 1981, 17, 494-504.

(55) Galstyan, V.; Pak, O. S.; Stone, H. A. A Note on the Breathing Mode of an Elastic Sphere in Newtonian and Complex Fluids. Phys. Fluids 2015, 27, 32001.

(56) Girard, A.; Gehan, H.; Mermet, A.; Bonnet, C.; Lermé, J.; Berthelot, A.; Cottancin, E.; Crut, A.; Margueritat, J. Acoustic Mode Hybridization in a Single Dimer of Gold Nanoparticles. Nano Lett. 2018, acs.nanolett.8b01072.

(57) Sherry, L. J.; Chang, S.-H.; Schatz, G. C.; Van Duyne, R. P.; Wiley, B. J.; Xia, Y. Localized Surface 
Plasmon Resonance Spectroscopy of Single Silver Nanocubes. Nano Lett. 2005, 5, 2034-2038.

(58) Zhang, S.; Bao, K.; Halas, N. J.; Xu, H.; Nordlander, P. Substrate-Induced Fano Resonances of a Plasmonic Nanocube: A Route to Increased-Sensitivity Localized Surface Plasmon Resonance Sensors Revealed. Nano Lett. 2011, 11, 1657-1663.

(59) Habteyes, T. G.; Dhuey, S.; Wood, E.; Gargas, D.; Cabrini, S.; Schuck, P. J.; Alivisatos, A. P.; Leone, S. R. Metallic Adhesion Layer Induced Plasmon Damping and Molecular Linker as a Nondamping Alternative. ACS Nano 2012, 6, 5702-5709.

(60) Huang, J.-S.; Callegari, V.; Geisler, P.; Brüning, C.; Kern, J.; Prangsma, J. C.; Wu, X.; Feichtner, T.; Ziegler, J.; Weinmann, P.; et al. Atomically Flat Single-Crystalline Gold Nanostructures for Plasmonic Nanocircuitry. Nat. Commun. 2010, 1, 150.

(61) Chen, K.-P.; Drachev, V. P.; Borneman, J. D.; Kildishev, A. V.; Shalaev, V. M. Drude Relaxation Rate in Grained Gold Nanoantennas. Nano Lett. 2010, 10, 916-922.

(62) Kelf, T. A.; Tanaka, Y.; Matsuda, O.; Larsson, E. M.; Sutherland, D. S.; Wright, O. B. Ultrafast Vibrations of Gold Nanorings. Nano Lett. 2011, 11, 3893-3898. 
a
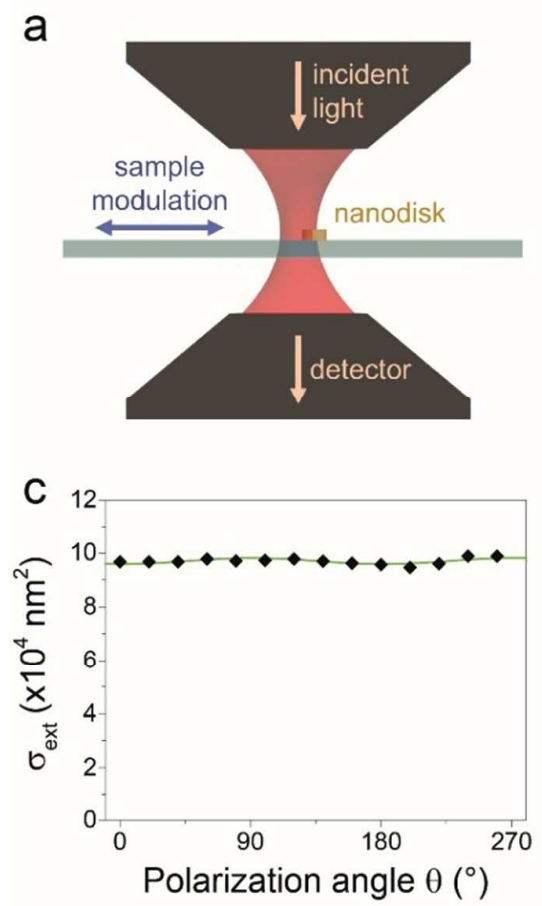

e

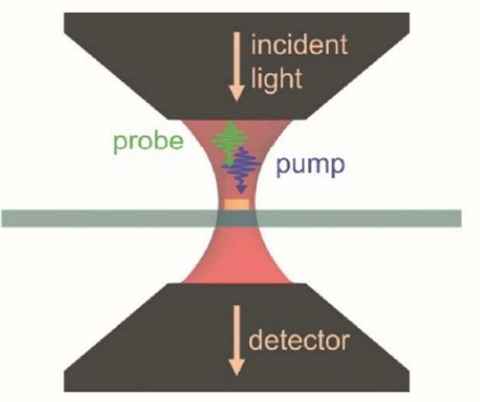

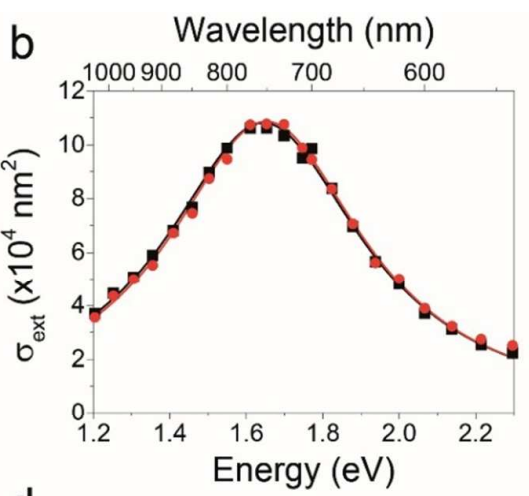
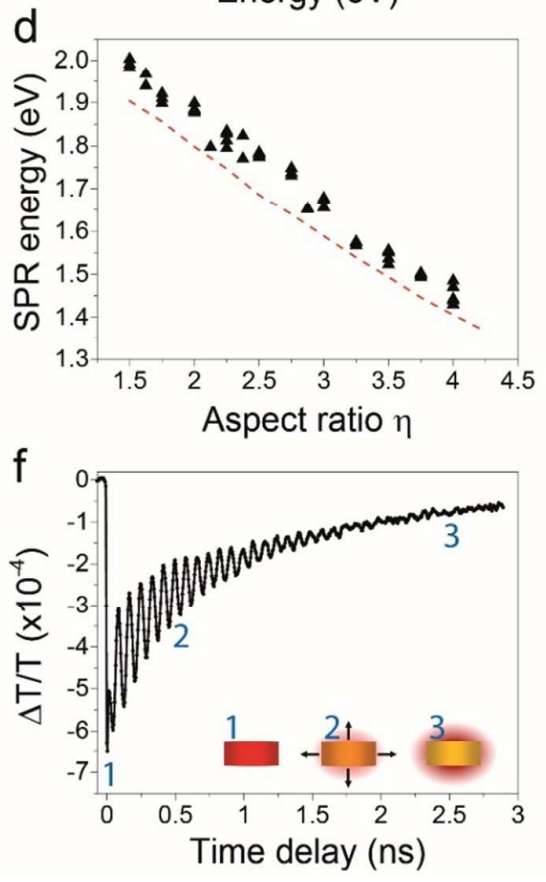

Figure 1. Linear and time-resolved optical experiments on individual gold NDs lithographed on a sapphire substrate. a) Principle of SMS experiments. b) Quantitative extinction spectrum $\sigma_{\text {ext }}$ of a ND ( $h=40 \mathrm{~nm}, \eta=3$ ), measured for the two orthogonal light polarizations respectively yielding maximal (black symbols) and minimal (red symbols) extinction at $\lambda=805 \mathrm{~nm}$ wavelength. Lorentzian fits of the spectra are shown as solid lines. c) Measured polarization dependence of $\sigma_{\text {ext }}$ at $\lambda=805 \mathrm{~nm}$ (black symbols) and fit with $f(\theta)=A+B \sin ^{2}\left(\theta+\theta_{0}\right)$ (green line). The very weak polarization dependences observed in panels b) and c) indicate that the investigated ND is quasi-circular. d) Aspect ratio dependence of the SPR spectral position measured for quasi-circular NDs (black triangles, each one corresponding to a single ND). The dashed red line shows the result of optical FEM calculations assuming a perfect cylindrical shape for the NDs and including their inhomogeneous environment. e) Principle of time-resolved experiments. f) Time-resolved relative transmission change, $\Delta T / T$, measured on a ND ( $h=40 \mathrm{~nm}, \eta=3.75)$ using $\lambda_{\mathrm{pp}}=475 \mathrm{~nm}$ and $\lambda_{\mathrm{pr}}=950 \mathrm{~nm}$ pump and probe wavelengths, showing signatures of ND excitation and internal thermalization (process 1 ), acoustic vibration (process 2 ) and cooling (process 3 ). 

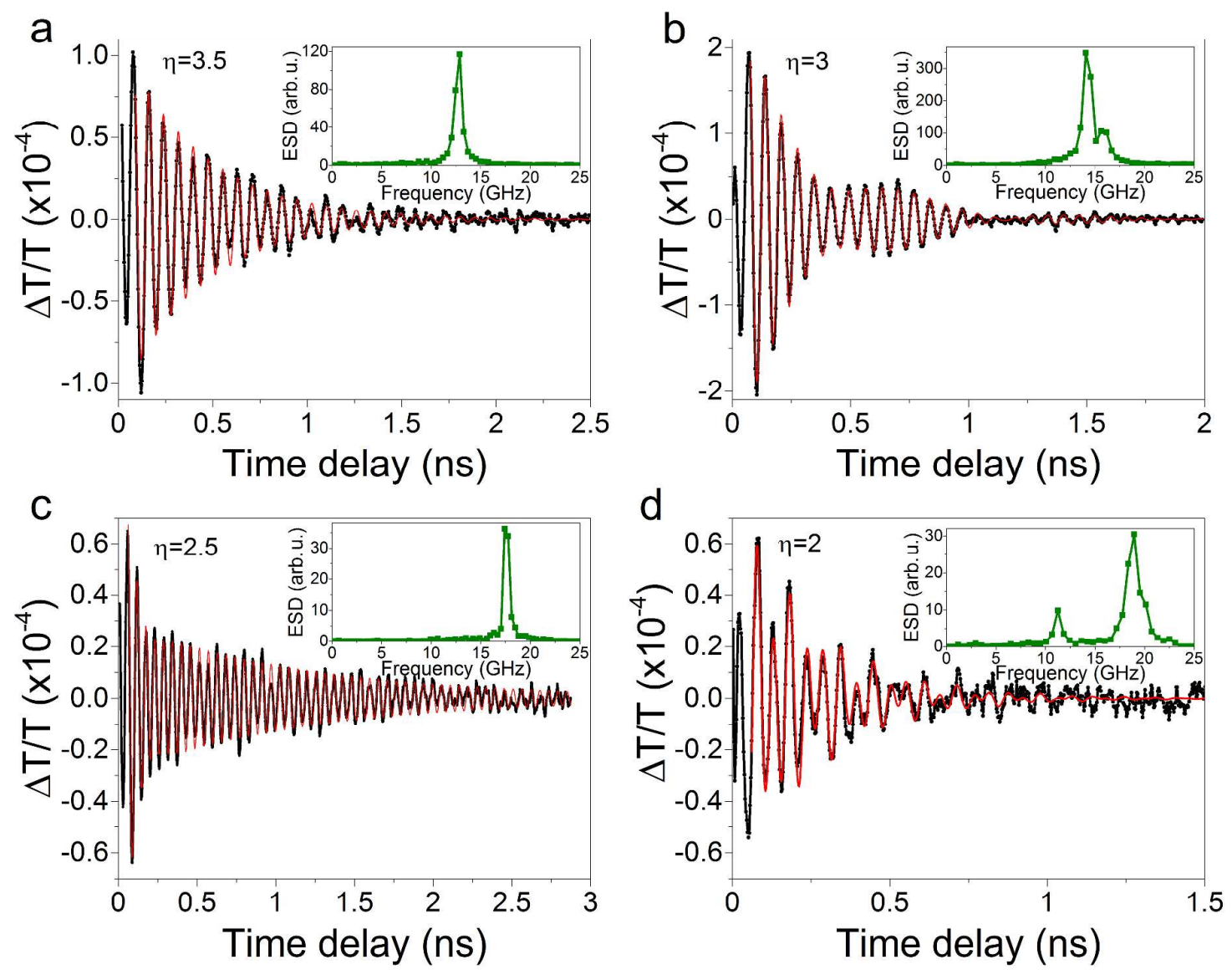

Figure 2. Oscillating components of representative pump-probe transmission changes $\Delta T / T$, measured for $h=40 \mathrm{~nm}$ individual gold NDs with $\eta=3.5$ (a, using $\lambda_{\mathrm{pp}}=475 \mathrm{~nm}$ and $\lambda_{\mathrm{pr}}=950 \mathrm{~nm}$ pump and probe wavelengths), 3 (b, $\lambda_{\mathrm{pp}}=420 \mathrm{~nm}$ and $\lambda_{\mathrm{pr}}=840 \mathrm{~nm}$ ), 2.5 (c, $\lambda_{\mathrm{pp}}=400 \mathrm{~nm}$ and $\lambda_{\mathrm{pr}}=800 \mathrm{~nm}$ ) and 2 (d, $\lambda_{\mathrm{pp}}=820 \mathrm{~nm}$ and $\lambda_{\mathrm{pr}}=600 \mathrm{~nm}$ ) (black lines). The red lines correspond to fits with one or two damped sinusoids (see main text). The energy spectral densities (ESD) of the measured oscillating components are shown in the insets. 


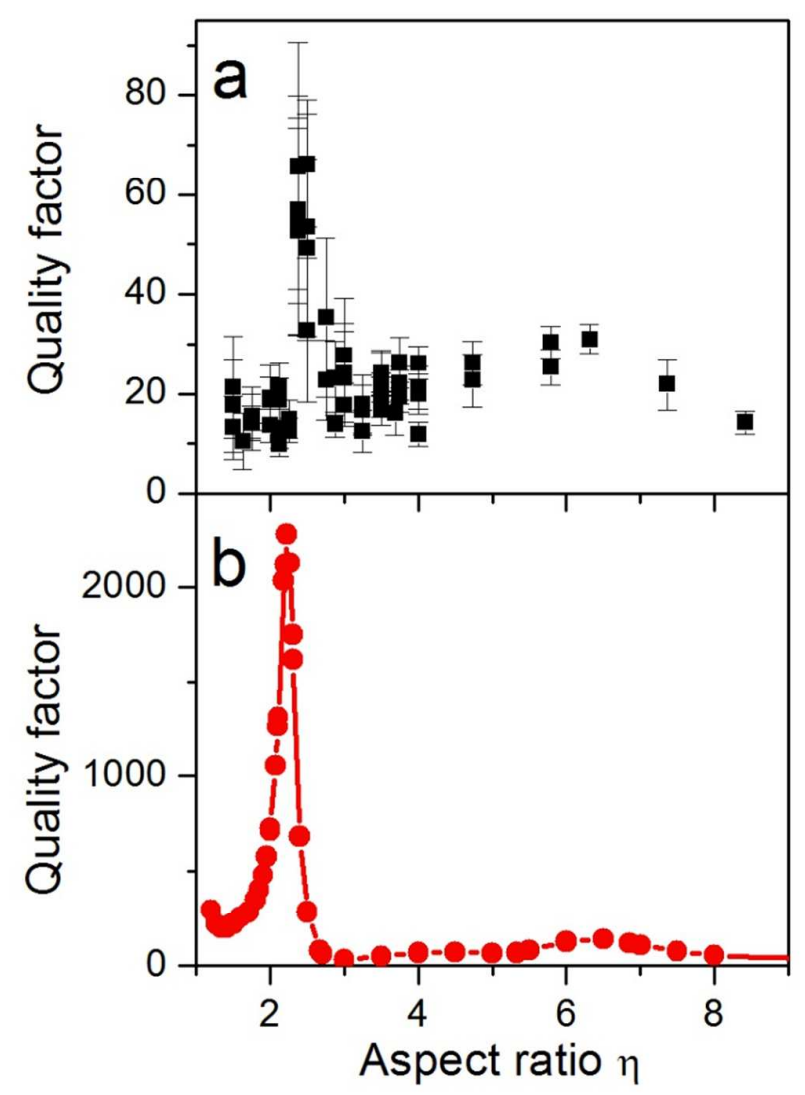

Figure 3. Highest quality factor a) of the experimentally detected vibrational modes (each dot corresponds to a single ND measurement) and b) of the FEM-computed modes as a function of ND aspect ratio $\eta$. 


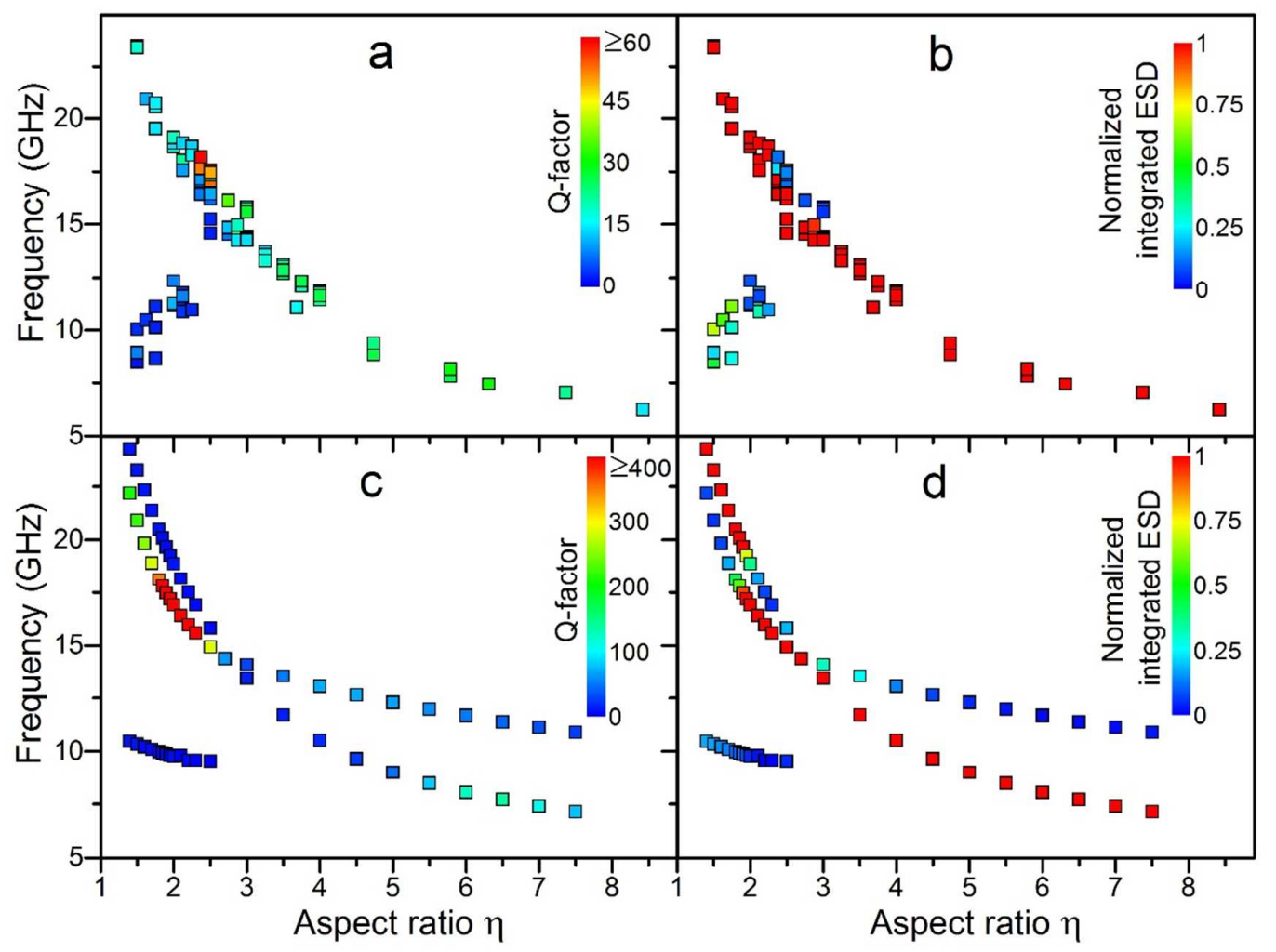

Figure 4. a-b) Experimental results: morphology dependences of the frequencies, quality factors (a) and normalized integrated ESD (b, reflecting the efficiencies with which each mode is excited and detected) of the vibrational modes detected in time-resolved experiments on $h=40 \mathrm{~nm}$ NDs. Measurements on $\mathrm{h}=20 \mathrm{~nm}$ NDs were also included in these plots, the measured frequencies being divided by 2 as they are predicted to scale as $1 / \mathrm{h}$ for given ND aspect ratio. c-d) Results of FEM simulations: computed frequencies, quality factors (c) and normalized integrated ESD (d, reflecting excitation efficiency) of the vibrational modes detected in the simulations. 

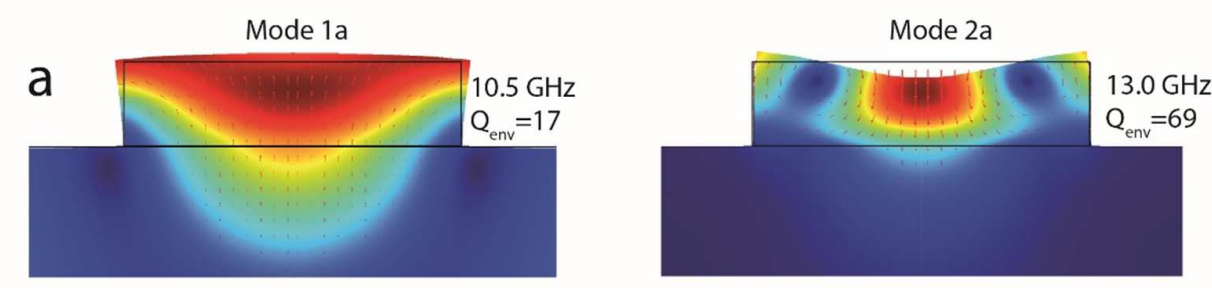

Displacement

(in phase component)
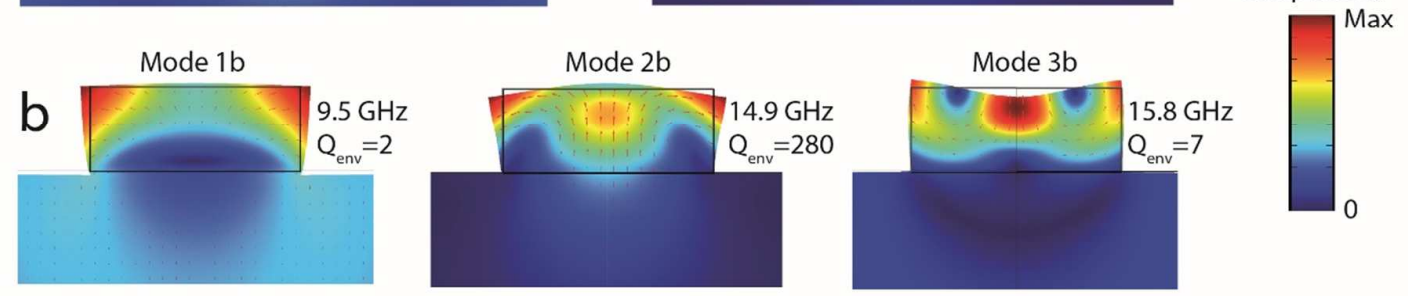

Figure 5. FEM-computed displacement profiles (vertical slices containing the ND center) of relevant vibrational modes of $a h=40 \mathrm{~nm}$ gold ND on a sapphire substrate for a) $\eta=4$ and $b) \eta=2.5$. The displacement component in phase with the excitation is plotted, with its amplitude color-coded. 
TOC
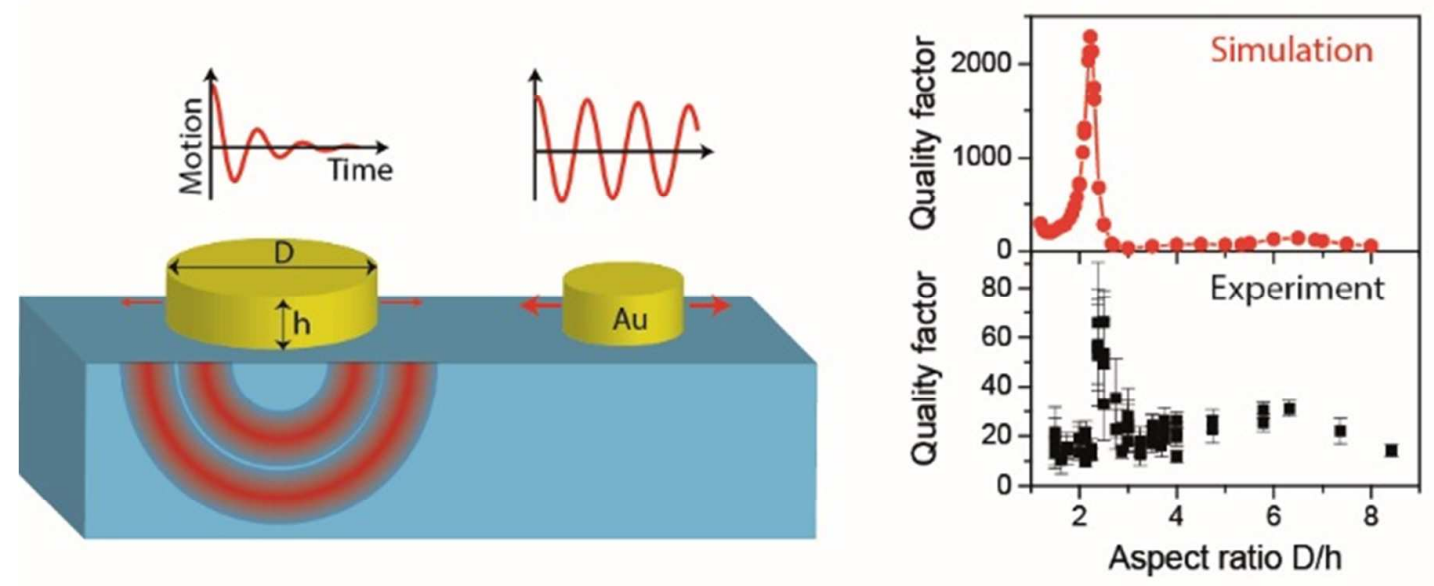